\title{
Original Syricte \\ Percutaneous drainage may not be necessary in a significant subset of patients with complicated liver abscess
}

\author{
Bishnu Saptarshi ${ }^{1}$, Partha Sarathi Patra ${ }^{2}$, Surender \\ Sultania ${ }^{2}$, Avik Sarkar ${ }^{3}$, Jaba Ranjan Hembram ${ }^{3}$, Gopal \\ Krishna Dhali ${ }^{2}$
}

\section{ABSTRACT}

Departments of ${ }^{1}$ Hepatology, ${ }^{2}$ Gastroenterology and ${ }^{3}$ GI Radiology, School of Digestive \& Liver Diseases, Institute of Post Graduate Medical Education \& Research, Kolkata.

Correspondence: Partha Sarathi Patra Email:pratim.ptr@gmail.com

Background: Clinical features and outcomes of liver abscess are ever changing, even in South Asia, where parasitic infections are common. It is important to devise management strategies to suit the changing needs. We wanted to capture this emerging spectrum in "real life" through assessment of clinical features, management strategies and outcomes of a cohort of treated liver abscess patients at a single center.

Methods: A retrospective study of patients admitted to a referral institution between February 2010 and June 2014 was undertaken. Patients with liver abscess who have received prior treatment in another hospital, and have then been referred for a complicated disease course were studied; their demographics, clinical presentations, evolution and outcomes were analyzed.

Results: A total of 154 patients [males $109,70.78 \%$ ), mean age $43.71 \pm 16.49$ years] were included in this study. Alcohol use was the most common (23.38\%) predisposing factor, followed by biliary obstruction (20.78\%) and diabetes (11.04\%). Most common presenting features were fever, pain abdomen and jaundice [92.9\%, 86.4\% and 20.8\% patients respectively]. Majority of patients (58.4\%) had a solitary liver abscess; right lobe being the commonest site(61\%). Median abscess volume was $180 \mathrm{ml}$ (Range 7-1524 $\mathrm{mL})$; abscess rupture occurred in $21(13.6 \%)$ cases, most commonlyin subdiaphragmatic location. Overall, abscess drainage was required in 97 $(63 \%)$ patients [single time aspiration $15(15.5 \%)$, percutaneous indwelling catheter $80(82.8 \%)$, surgical drainage $2(2.1 \%)]$. Patients who required percutaneous drainage were mostly males, had higher abscess volumes and were more frequently alcohol users and diabetics. Six (3.9\%) patients died in the study period.

Conclusion: While large abscess, alcoholism and diabetes were associated with increased need for catheter drainage, $37 \%$ can be treated by drug therapy alone. Careful selection of subjects for drainage may help in decreasing the mortality rate in this benign condition.

KEYWORDS: Liver abscess; percutaneous drainage. 


\section{Introduction}

Liver abscess refers to an infected space-occupying lesion in the hepatic parenchyma. It remains an important clinical problem with a significant mortality rate in both developing and developed countries. It could result as a complication of various intraabdominal infections including that of biliary tract; by hematogenous spread via portal vein from the gastrointestinal tract; or, may develop after traumatic injury to the liver. The two most common types of liver abscesses are pyogenic and amoebic. Pyogenic liver abscess has a reported incidence of 20 per $1,00,000$ hospital admissions in a western population. ${ }^{1}$ Ascending biliary tract infection because of extrahepatic biliary obstruction is the most commonly identifiable cause of pyogenic liver abscess. Biliary obstruction in this setting may be in the form of malignant diseases, as in Western countries, or may be due to predominantly gallstone disease and hepatolithiasis in Asia. ${ }^{1,2}$ Amoebic liver abscesses are mostly seen in tropical countries where Entamoeba histolytica is endemic and is more prevalent in individuals with defective cell mediated immunity. ${ }^{3}$ Clinical presentations of amoebic and pyogenic liver abscesses share many similarities and can confound the clinician. ${ }^{4}$ The standard treatment of liver abscess is the use of appropriate antibiotics and supportive care. Recent literature on percutaneous drainage procedure have shown a favorable outcome with less average length of stay in hospital compared to conservative mode of treatment. ${ }^{5}$ The aim of this present study was to evaluate the clinical profile and scenario in which liver abscess occurs, the role of percutaneous drainage in management of liver abscess and outcomes in these patients.

\section{Methods}

This study was undertaken at School of Digestive \& Liver Diseases, IPGME \& R, Kolkata, a referral Gastroenterology and Hepatology center in eastern India. A retrospective record review of patients admitted between February 2010 and June 2014 was undertaken to identify patients who had been admitted with the diagnosis of liver abscess. Data pertaining to demographics (age, sex), clinical presentations (duration of symptoms, presence of fever/pain abdomen/jaundice/shortness of breath/abdominal swelling), past history (risk factors for liver abscess, alcohol use, chronic diseases like diabetes, prior history of liver abscess) blood biochemistry and hematology data (liver function test, hemoglobin, total leucocyte count, platelet count, creatinine), radiological findings (number, location and total volume of abscess(es), presence of abscess rupture and it's site), microbiological findings (Gram stain findings, growth on culture, antibiotic sensitivity), medications used (fluoroquinolones and/or metronidazole), drainage procedures performed (single time aspiration versus pigtail catheter drainage- number, size of pigtail catheter(s) used, duration of pigtail drainage) and outcomes (duration of stay, recovery, requirement of surgery, death) during hospital stay, were retrieved from electronic medical record database. These were reviewed with respect to completeness and transferred to a predesigned Microsoft Office Excel worksheet. Measures were taken to maintain privacy and confidentiality of patient data by anonymization. Final data sheet was subjected to analysis by statistical software Epi Info 7 (Centre for Disease Control, Atlanta, USA). Descriptive statistical terms like percentage, ratio and proportion were used for categorical variables, and mean, median, standard deviation and percentiles were used for quantitative analysis. The research protocol was approved by the institutional review board.

\section{Results}

A total of 177 patient records were retrieved from Electronic Medical Records responding to the search query for "liver abscess". Out of these, 18 records were incomplete or diagnosis could not be confirmed, and were not included in subsequent analysis. Four patients also had multiple admissions ( 3 admissions for 1 patient and 2 admissions for 3 patients) and were considered as a single admission. The remaining 154 patient recorded were considered for inclusion in this study and for further analysis. 


\section{Demographics and risk factors}

The average age of patients admitted with liver abscess in our study was $43.7 \pm 16.5$ years, while males comprised $70.8 \%(n=109)$ of our study population. Possible risk factors identified were alcohol use in $36(23.4 \%)$ patients, biliary obstruction in $32(20.8 \%)$ and diabetes in $17(11 \%)$ patients. No risk factors could be attributed in 61 (39.6\%) patients.

\section{Clinical presentation}

Mean duration of illness before admission was $24.8 \pm 22$ days. The commonest presenting symptom was fever in $143(92.86 \%)$ patients, followed by pain abdomen, jaundice, shortness of breath and abdominal swelling in $133(86.36 \%), 32$ (20.78\%), 18 (11.69\%) and $16(10.39 \%)$ patients respectively. Five $(3.29 \%)$ patients had a prior history of having suffered from liver abscess.

\section{Laboratory dataand radiological findings (Table 1)}

Majority of patients $(90,58.44 \%)$ had a solitary liver abscess; right lobe being the commonest location (94, $61.04 \%$. Median abscess volume was $180 \mathrm{ml}$ (7-1524 $\mathrm{mL})$; abscess rupture occurred in 21 (13.64\%) cases, most commonly to subdiaphragmatic location $(n=12,57.1 \%)$.

\section{Treatment received}

One hundred and fourteen patients (74.03\%) received treatment with fluoroquinolones while 107 (69.48\%) received metronidazole. Abscess drainage was required in 95 (61.69\%) patients; 15 (9.74\%) patients underwent ultrasound (US) guided single time aspiration while $80(90.26 \%)$ patients required US guided placement of indwelling pigtail catheter in abscess cavity. The mean duration of indwelling catheter drainage required was $5.66 \pm 3.79$ days. Requirement of percutaneous drainage was significantly associated with male gender, ethanol consumption, diabetes, higher abscess volume, and lower serum bilirubin values (Table 2). Surgical intervention was done in $2(1.30 \%)$ patients. Mean duration of hospitalization was $8.06 \pm 4.73$ days. Mortality rates among liver abscess patients stood at $3.9 \%(6 / 152)$ in this study.

\section{Microbiological examination}

Aspirated pus from liver abscess was sent for gram stain and culture in 35 patients; in 12 (34.29\%) cases, causative organisms could be identified asKlebsiella, E.coli and Pseudomonas in 6, 5 and 1 cases respectively.

\section{Subgroup: Pyogenic liver abscess}

Of the 32 patients with pyogenic liver abscess, 12 $(37.50 \%)$ were males and the average age at presentation was $45.59 \pm 14.71$ years. These patients with pyogenic liver abscess were symptomatic for a mean duration of $23.56 \pm 18$ days before presentation. The commonest presentations in this subgroup were fever, pain abdomen and jaundice, being present in $93.75 \%, 78.13 \%$ and $59.38 \%$ patients respectively. The most frequent among

Table 1: Depicting laboratory data and ultrasound findings for the study patients

\begin{tabular}{l|l|l|l} 
Abscess number & Laboratory parameters \\
\hline One & $90(58.44 \%)$ & Serum bilirubin $(\mathrm{mg} / \mathrm{dL})$ & $0.8(0.1-34.3)$ \\
\hline Two & $26(14.29 \%)$ & SGPT $(\mathrm{IU} / \mathrm{mL})$ & $38(10-430)$ \\
\hline Multiple & $34(27.27 \%)$ & SGOT $(\mathrm{IU} / \mathrm{mL})$ & $51(13-680)$ \\
\hline Abscess location & & ALP $(\mathrm{U} / \mathrm{L})$ & $322(34-1056)$ \\
\hline Right lobe & $94(61.04 \%)$ & Serum albumin $(\mathrm{g} / \mathrm{dL})$ & $2.6(1.5-4.1)$ \\
\hline Left lobe & $26(16.88 \%)$ & INR & $1.33(1-4.8)$ \\
\hline Both lobes & $34(22.08 \%)$ & Hemoglobin $(\mathrm{gm} / \mathrm{dL})$ & $10.08 \pm 2.05$ \\
\hline Abscess volume (mililitre) & $285.98 \pm 280.03$ & Total leukocyte count $\left(10^{3} / \mathrm{mL}\right)$ & $15.79 \pm 8.12$ \\
\hline Abscess ruptured & $21(13.64 \%)$ & Platelet count $\left(10^{3} / \mathrm{mL}\right)$ & $274.35 \pm 131.73$
\end{tabular}


Table 2: Characteristics of patients with liver abscess classified according to requirement of percutaneous drainage

\begin{tabular}{|c|c|c|c|c|}
\hline & & \multicolumn{3}{|c|}{ Percutaneous drainage required } \\
\hline & & No $(n=59)$ & Yes $(\mathrm{n}=95)$ & p value \\
\hline Age (years) Mean \pm SD & & $44 \pm 18$ & $44 \pm 16$ & 0.58 \\
\hline \multirow[t]{2}{*}{ Sex } & Male & $30(50.85 \%)$ & $79(83.16 \%)$ & \multirow{2}{*}{.0001} \\
\hline & Female & $29(49.15 \%)$ & $16(16.84 \%)$ & \\
\hline \multirow[t]{3}{*}{ Abscess Location } & Right lobe & $33(55.93 \%)$ & $61(64.21 \%)$ & \multirow{3}{*}{.159} \\
\hline & Left lobe & $8(13.56 \%)$ & $18(18.95 \%)$ & \\
\hline & Both lobes & $18(30.51 \%)$ & $16(16.84 \%)$ & \\
\hline \multicolumn{2}{|l|}{ Abscess volume $(\mathrm{mL})$ Median, range } & $58(7-525)$ & $286(70-1524)$ & .0001 \\
\hline \multicolumn{2}{|l|}{ Abscess rupture $(\mathrm{n}, \%)$} & $2(3.51 \%)$ & $19(19.59 \%)$ & .004 \\
\hline \multirow[t]{3}{*}{ Presence of risk factors } & Ethanol & $6(10.17 \%)$ & $29(30.53 \%)$ & \multirow{3}{*}{.018} \\
\hline & Biliary obstruction & $25(42.37 \%)$ & $7(7.37 \%)$ & \\
\hline & Diabetes & $4(6.78 \%)$ & $14(14.73 \%)$ & \\
\hline \multicolumn{2}{|c|}{ Total symptomatic period (days) Median, range } & $25(9-162)$ & $27(9-110)$ & .330 \\
\hline \multicolumn{2}{|l|}{ Mortality $(\mathrm{n}, \%)$} & $0(0 \%)$ & $6(6.32 \%)$ & .05 \\
\hline \multicolumn{2}{|l|}{ Serum bilirubin (mg/dl) Median, range } & $1.20(.1-34.30)$ & $0.72(.5-18.50)$ & 0.024 \\
\hline \multicolumn{2}{|l|}{ INR Median, range } & $1.34(1-4.80)$ & $1.32(1-2.30)$ & .370 \\
\hline
\end{tabular}

the causes of biliary obstruction were choledocholithiasis [15 (44.12\%)], biliary malignancy [7 (20.59\%)] and benign biliary stricture [4(11.76\%)], followed by recurrent pyogenic cholangitis [3 $(8.82 \%)$ ], hepaticojejunostomy stricture $[2(5.88 \%)]$ and 1 case $(2.94 \%)$ each of biliary ascariasis, iatrogenic bile duct injury and combined choledocholithiasis with biliary malignancy.

Fifty percent $(n=16)$ patients had multiple abscesses while $13(40.63 \%)$ had a single abscess. The average abscess volume was $118.90 \pm 80.00 \mathrm{~mL}$ and rupture of abscess occurred in only 2 out of 32 cases. Seven out of 32 patients $(21.88 \%)$ with pyogenic abscess required percutaneous drainage, with 3 patients undergoing in dwelling catheter insertion, 2 requiring single time percutaneous aspiration and 2 patients undergoing both modalities.

\section{Discussion}

Liver abscess is an important clinical problem in a tropical country like India. The two commonest types of liver abscess (pyogenic and amoebic) are difficult to differentiate clinically and require further investigations and/or invasive procedures to establish the diagnosis; this is necessary as they have different modalities of treatment. Amoebic liver abscess have been reported more commonly at younger ages (40.5-43.64), ${ }^{6,7}$ while pyogenic liver abscess has been found to be more common at more than 60 years of age in Western population. ${ }^{89}$ The mean age of patients in our study (43.71 years) corroborates data from previous Indian studies, ${ }^{6,7}$ and is inconsistent with that reported in a similar retrospective study from the United Kingdom. ${ }^{10}$

Along with biliary obstruction, with respectto risk factors, cryptogenic mechanisms have been held responsible for most liver abscesses, ${ }^{10,11,12}$ In our study also, the underlying predisposing factor could not be identified in two fifths of cases. In a study from Hong Kong, ${ }^{13}$ ERCP findings in patients of pyogenic liver abscess revealed no abnormality in $38 \%$ cases and choledocholithiasis in $22 \%$ cases, while in our study, choledocholithiasis was present in $44 \%$ of patients with biliary abnormalities, followed by malignant biliary stricture in $21 \%$.

In a study from India, pain abdomen, fever and anorexia have been reported by Ghosh et al to be the commonest (93-99\%) presentation of liver abscess; ${ }^{14}$ this is also consistent with the findings of our study with fever present in $93 \%$ and pain abdomen in $86 \%$ patients. 
They have also reported right lobe of liver to be the site of abscess in $71 \%$ cases, a figure slightly greater than our own $(61 \%)$. Also, in our study, the incidence of rupture was found to be $13.64 \%$, which is higher than previously reported frequency of $3 \%{ }^{15}$ This can be since we have studied patients who were referred to us for incomplete resolution of symptoms after initiation of treatment elsewhere.

Decision regarding percutaneous aspiration of pus is often contentious and requires assessment of clinical status in addition to imaging modalities. A recent randomized trial from India has revealed that in the management of uncomplicated, solitary, right lobe amoebic liver abscess of 5 to $10 \mathrm{~cm}$, percutaneous needle aspiration along with metronidazole was no better than metronidazole alone with regards to duration of hospitalization, frequency of abscess rupture and resolution of pain and fever. ${ }^{16}$ Another recent study has underlined the better outcomes of using percutaneous catheter drainage over percutaneous needle aspiration in management of large $(>10 \mathrm{~cm}$ diameter) amoebic liver abscesses. ${ }^{17}$ In our study, we identified that male patients and those with ethanol consumption, diabetes, higher abscess volume values were more likely to require percutaneous drainage of liver abscess. We also demonstrated that overall, abscess drainage was required in 97 (63\%) patients, thus $37 \%$ patients even with complicated disease course were treated by medical management alone.

\section{Conclusion}

This study demonstrated that while large abscess, alcoholism and diabetes were associated with increased need for catheter drainage, $37 \%$ could be treated by drug therapy alone. Careful selection of subjects for drainage might decrease the mortalityrate in this benign condition.

\section{References}

1. Huang CJ, Pitt HA, Lipsett PA et al. Pyogenic hepatic abscess: changing trends over 42 years. Ann Surg. 1996;223:600-9.
2. Chu KM, Fan ST, Lai EC et al. Pyogenic liver abscess: an audit of experience over the last decade. Arch Surg. 1996;131:148-52.

3. Sharma MP, Ahuja V. Management of amoebic and pyogenic liver abscess. Indian J Gastroenterol. 2001;20, supplement C33-36.

4. Rustgi AK, Richter JM. Pyogenic and Amoebic liver abscess. Med Clin North Am. 1989;73:847-58.

5. Yu SC, Ho SS, Lau WY et al.Treatment of pyogenic liver abscess: prospective randomized comparison of catheter drainage and needle aspiration. Hepatology. 2004;39(4):932-38.

6. Sharma N, Sharma A, Varma Set al.Amoebic liver abscess in the medical emergency of a North Indian hospital. BMC Res Notes. 2010;3:21-24.

7. Mukhopadhyay M,SahaAK, Sarkar A et al. Amoebic liver abscess: presentation and complications. Indian J Surg. 2010;72(1):37-41.

8. Heneghan HM,Healy NA,Martinet ST.Modern management of pyogenic hepatic abscess: a case series and review of the literature. BMC Res. Notes2011;4:80-87.

9. Mohsen AH, Green ST, Reed RC et al. Liver abscess in adults: ten years experience in a UK centre. $Q J \mathrm{Med}$. 2002;95(12):797-802.

10. Bertel CK, Van Heeden JA, Sheedy PF II. Treatment of pyogenic hepatic abscesses: surgical vs PCD. Arch Surg. 1986;121:554-8.

11. Cohen JL, Martin MF, Rossi LR et al. Liver abscess: the need for complete gastrointestinal evaluation. Arch Surg. 1989;124:561-4.

12. Greenstein AJ, Lowenthal D, Hammer GS et al. Continuing changing patterns of disease in pyogenic liver abscess: a study of 38 patients. Am J Gastroenterol. 1984;79:217-26.

13. Lok KH, Li KF, Li KK et al. Pyogenic liver abscess: clinical profile, microbiological characteristics, and management in a Hong Kong hospital. J Microbiol Immunol Infect. 2008;41:483-490.

14. Ghosh S, Sharma S, Gadpayle AK et al. Clinical, Laboratory, and Management Profile in Patients of Liver Abscess from Northern India. Indian. J Trop Med. 2014: $1-8$.

15. Ramani A, Ramani R, Shivananda PG. Amoebic Liver Abscess. A Prospective Study of 200 Cases in A Rural Referral Hospital In South India. Bahrain Medical Bulletin. 1995;17(4).

16. Bammigatti C, Ramasubramanian NS, Kadhiravan Tet al. Percutaneous needle aspiration in uncomplicated amebic 
liver abscess: a randomized trial. Trop Doct. 2013;43(1): 19-22.

17. Gupta SS, Singh O, Sabharwal G et al. Catheter drainage versus needle aspiration in management of large $(>10$ $\mathrm{cm}$ diameter) amoebic liver abscesses. ANZ Journal of Surgery. 2011;81:547-551. 